\title{
Hecke Functors and the Equivariant Dold-Thom Theorem
}

Dedicated to Professor Minoru Nakaoka on his 60-th birthday

By

\section{Goro NISHIDA*}

\section{§1. Introduction}

We recall that the Dold-Thom theorem [3] asserts that the weak homotopy type cf a topological abelian group is determined by its homotopy groups, and hence the homotopy category of topological abelian groups with respect to weak equivalences is equivalent to the category of graded abelian groups.

In this note we consider the equivariant version. We restrict ourself to the case of finite group actions. Let $G$ be a finite group, and let $k$ be a commutative ring with unit. A topological $k[G]-$ module is a topological abelian group $M$ as well as a $k[G]$-module such that the bilinear map

$$
k[G] \times M \longrightarrow M
$$

is continuous, where $k$ is given the discrete topology. We denote by $\boldsymbol{M}_{k[G]}$ the category cf topological $k[G]$-modules and continuous $k[G]$ homomorphisms. A morphism $f: M \rightarrow M^{\prime}$ is called a weak equivalence if the induced homomorphism

$$
f_{*}: \omega_{n}(M) \longrightarrow \omega_{n}\left(M^{\prime}\right)
$$

is an isomorphism for any $\mathrm{n} \geq 0$, where $\omega_{n}$ denotes the Bredon homotopy group [1]. Then the purpose of this note is to determine the homotopy category $\mathrm{Ho}\left(\boldsymbol{M}_{k[G]}\right)$ with respect to weak equivalences in terms of Hecke functors (Theorem 4.1). Moreover if the ring $k$ is good for given $G$, then one can show that $\mathrm{Ho}_{\left(\boldsymbol{M}_{\left.k_{-} G\right]}\right)}$ is equivalent to the category of the graded Hecke functors (Theorem 4.5).

\footnotetext{
Communicated by N. Shimada, September 11, 1982.

* Department of Mathematics, Kyoto University, Kyoto 606, Japan.
} 


\section{§ 2. Hecke Functors}

Let $G$ be a finite group and let $S$ be a finite $G$-set. Let $\mathbb{Z}[S]$ denote the free abelian group generated by $S$ with the obvious $G-$ action. Let $\boldsymbol{L}^{G}$ denote the category whose objects are all finite $G$ sets and $\boldsymbol{L}^{G}(S, T)=\operatorname{Hom}_{\boldsymbol{Z}[G]}(\boldsymbol{Z}[S], \boldsymbol{Z}[T])$. Let $k$ be a commutative ring with unit and let $k$-Mod be the category of $k$-modules.

Definition 2.1. A Hecke functor over $k$ is an additive contravariant functor

$$
H: L^{G} \longrightarrow k-\operatorname{Mod}
$$

which sends a finite sum in $\boldsymbol{L}^{G}$ into a finite product in $k$-Mod. A natural transformation $\alpha: H \rightarrow H^{\prime}$ is called a morphism of Hecke functors. The category of Hecke functors over $k$ is denoted by $\mathbf{H e c}_{k}^{G}$.

Let $\boldsymbol{L}_{\infty}^{G}$ be the category obtained from $\boldsymbol{L}_{G}$ by deleting the finiteness condition. For each $G$-set $S$ choose an isomorphism $S \cong \prod S_{\lambda}, S_{\lambda}$ is finite. Let $H$ be a Hecke functor, then putting $H(S)=\prod H\left(S_{\lambda}\right)$ we obtain an additive contravariant functor $H: \boldsymbol{L}_{\infty}^{G} \rightarrow k$-Mod which sends a sum into a product. Therefore in the following by a Hecke functor we mean such a functor $\boldsymbol{L}_{\infty}^{G} \rightarrow k$-Mod.

Next let $\boldsymbol{L}_{k}^{G}$ be the category in which $\mathrm{Ob}\left(\boldsymbol{L}_{k}^{G}\right)=\mathrm{Ob}\left(\boldsymbol{L}_{\infty}^{G}\right)$ and $\boldsymbol{L}_{k}^{G}(S, T)=\operatorname{Hom}_{k[G]}(k[S], k[T])$. Note that $\operatorname{Hom}_{k[G]}(k[\mathrm{~S}], k[\mathrm{~T}]) \cong$ $\operatorname{Hom}_{\boldsymbol{Z}[G]}(\boldsymbol{Z}[S], \boldsymbol{Z}[T]) \otimes k$, and there is an obvious functor $\boldsymbol{L}_{\infty}^{G} \rightarrow \boldsymbol{L}_{k}^{G}$. Therefore a Hecke functor $H: \boldsymbol{L}_{\infty}^{G} \rightarrow k-$ Mod is uniquely factored as $\boldsymbol{L}_{\infty}^{G} \rightarrow \boldsymbol{L}_{k}^{G} \stackrel{\tilde{H}}{\longrightarrow} k$-Mod.

It is well known that a Hecke functor is a Mackey functor [4] in a canonical way and moreover the following is known.

Theorem 2.2. ([8] and [11]). A Mackey functor $M$ has a structure of a Hecke functor if and only if $M$ is cohomological.

For the definition of cohomological Mackey functors, see [5].

Let $V$ be a $k[G]$-module. Let $\bar{V}$ denote the Hecke functor represented by $V$, namely

$$
\bar{V}(S)=\operatorname{Hom}_{k[G]}(k[S], V) .
$$


Let $G / H$ be a coset. Then we have isomorphisms

$$
\operatorname{Hom}_{k[G]}(k[G / H], V) \cong \operatorname{Map}_{G}(G / H, V) \cong V^{H} \text {. }
$$

Where $V^{H}$ is the submodule of $\mathrm{H}$-fixed points.

Now we consider the abelian category $\mathbf{H e c}_{k}^{G}$. Then the following proposition is easily checked from definition.

Proposition 2.3. i) Let $H$ be a Hecke functor over $k$ and let $S$ be a G-set. Then there is a natural isomorphism

$$
\operatorname{Mor}(\overline{k[S]}, H) \cong H(S) \text {. }
$$

ii) Let $S$ be a G-set. Then $\overline{k[S]}$ is projective and for any Hecke functor $H$ there is a G-set $T$ and an epimorphism $\overline{k[T]} \rightarrow H$.

\section{§3. Topological $k[G]$-Modules}

Let $k$ be a commutative ring with unit as before. Let $R$ be a topological $k[G]$-module, namely, $R$ is a topological abelian $G$-group as well as a $k[G]$-module such that the bilinear map $k[G] \times R \rightarrow R$ is continuous. Let $X$ be a based $G$-space. Define a $G$-space $R \otimes X$ by $\left(\frac{1}{n} R^{n} \times X^{n}\right) / \sim$ where the equivalence relation is given by ( $\varphi_{*} a$, $x) \sim\left(a, \varphi^{*} x\right), \varphi: \boldsymbol{n} \rightarrow \boldsymbol{m}$ is a based set map, $\boldsymbol{n}=\{0,1, \cdots, n\}, a=\left(a_{1}\right.$, $\left.\cdots, a_{n}\right) \in R^{n}, x \in X^{m}=\operatorname{Map}_{*}(\boldsymbol{m}, X),\left(\varphi_{*} a\right)_{i}=\sum_{j \in \varphi^{-1}(i)} a_{j}$ and $\varphi^{*} x=x \circ \varphi$. Let $S$ be a $G$-set, then $k \otimes S_{+}$is identified with $k[S]$, and $Z \otimes X$ is the free abelian topological $G$-group generated by $X$.

Lemma 3.1. i) $R \otimes X$ is a topological $k[G]$-module.

ii) $(R \otimes X) \otimes Y \cong R \otimes(X \wedge Y)$ as topological $k[G]$-modules.

Proof. Define the product $(R \otimes X) \times(R \otimes X) \rightarrow R \otimes X$ by the juxtaposition which is clearly well-defined. Let $\iota: R \rightarrow R$ be the map given by $\iota(r)=-r$. Then the continuous map $\iota \otimes \mathrm{id}: R \otimes X \rightarrow R \otimes X$ gives the inverse homomorphism. A $k$-module structure is similarly defined. Define $g[(a, x)]=[(g a, g x)]$ for $g \in G$, then we have a linear $G$-action and this shows i). Next for $u=\left(x_{1}, \cdots, x_{m}\right) \in X^{m}$ and $y \in Y$, let $u \cdot y=\left(\left(x_{1}, y\right), \cdots,\left(x_{m}, y\right)\right) \in(X \times Y)^{m}$. Define $\lambda: \Perp\left(\Perp R^{m} \times\right.$ $\left.X^{m}\right)^{n} \times Y^{n} \rightarrow \Perp R^{k} \times(X \times Y)^{k}$ by $\lambda\left(\left(a_{1}, u_{1}\right), \cdots,\left(a_{n}, u_{n}\right) ; y_{1}, \cdots, y_{n}\right)=$ $\left(\left(a_{1}, \cdots, a_{n}\right) ;\left(u_{1} \cdot y_{1}, \cdots, u_{n} \cdot y_{n}\right)\right)$. Then $\lambda$ induces a well defined 
$G$-homeomorphism $(R \otimes X) \otimes Y \rightarrow R \otimes(X \wedge Y)$.

Lemma 3.2. Let $X$ be a G-space with the trivial G-action. Then for any subgroup $H \subset G$, there is a homeomorphism $(R \otimes X)^{H} \cong R^{H} \otimes X$.

Proof. An element $a=\left(a_{1}, \cdots, a_{n}\right) \in R^{n}$ is called non-degenerate if $a_{i} \neq 0$ for any $i$, and an element $x=\left(x_{1}, \cdots, x_{n}\right) \in X^{n}$ is called nondegenerate if $x_{i} \neq *$ (base point) for any $i$ and $x_{i} \neq x_{j}$ for any $i$ and $j$. It is easy to see that any element of $R \otimes X$ is represented by a non-degenerate $(a, x) \in R^{n} \times X^{n}$ uniquely up to the action of $\Sigma_{n}=$ Aut $\boldsymbol{n}$. Let $u=[(a, x)] \in R \otimes X$ be represented as above and suppose that $u \in(R \otimes X)^{H}$. Note that for any $h \in H$, ha and $h x$ are nondegenerate. Hence $h u=u$ implies that $(h a, h x) \sim(a, x)$. Therefore $h x=\sigma^{*}(x)$ and $\sigma_{*}(h a)=a$ for some $\sigma \in \Sigma_{n}$. Since $X$ is a trivial $G$ space, $h x=x$, and since $x$ is non-degenerate we see that $\sigma=e$ and $h a=a$. This shows that $\mathrm{a} \in\left(R^{H}\right)^{n}$ and we easily have a homeomorphism $(R \otimes X)^{H} \cong R^{H} \otimes X$.

We denote the category of topological $k[G]$-modules and continuous $k[G]$-homomorphisms by $\mathbb{M}_{k[G]}$. Let $R, Q \in \mathrm{Ob}\left(\mathbb{M}_{k[G]}\right)$ and let $f_{0}, f_{1}$ : $R \rightarrow Q$ be morphisms, then a homotopy of $f_{0}$ and $f_{1}$ in $M_{k[G]}$ is obviously defined and denoted by $f_{0} \sim_{\oplus} f_{1}$. We denote by $[R, Q]_{R[G]}^{\oplus}$ the homotopy set of morphisms. Let $X$ be a based $G$-space and let $f: X \rightarrow R$ be a based $G$-map. Then there is a unique morphism $\tilde{f}: k \otimes X \rightarrow R$ extending $f$. This defines a natural homomorphism

$$
\rho:[X, R]_{G} \longrightarrow[k \otimes X, R]_{k[G\rceil}^{\oplus}
$$

Then as is easily seen we have

Lemma 3.3. $\rho$ is an isomorphism.

Let $O^{G}$ be the category of $G$-orbits and $G$-maps. A contravariant functor $B: O^{G} \rightarrow$ Set is called a Bredon functor. One can regard $O^{G}$ as a subcategory of $\boldsymbol{L}^{G}$, and hence a Hecke functor determines the underlying Bredon functor. Let $X$ be a based $G$-space. Define a Bredon homotopy group [1] to be a Bredon functor $\omega_{n}(X)$ given by

$$
\omega_{n}(X)(G / H)=\pi_{n}\left(X^{H}\right) .
$$


For $n \geq 2$ this is clearly a module valued functor.

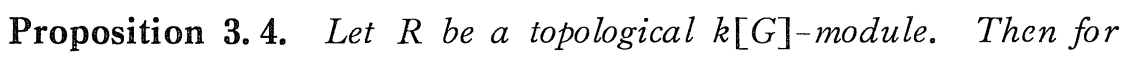
any $n$ the Bredon homolopy group $\omega_{n}(R)$ is a Hecke functor over $k$.

Proof. Recall that $\omega_{n}(R)(G / H)=\left[S^{n} \wedge\left(G / H_{+}\right), R\right]_{G}$. By Lemma 3.1, we have $k \otimes\left(S^{n} \wedge\left(G / H_{+}\right)\right) \cong k[G / H] \otimes S^{n}$. Then by Lemma 3.3 we have as isomorphism

$$
\omega_{n}(R)(G / H) \cong\left[k[G / H] \otimes S^{n}, R\right]_{k[G]}^{\oplus} .
$$

Let $\alpha \in \operatorname{Hom}_{k[G]}(k[G / K], k[G / H])$, then we easily have a homomorphism

$$
(\alpha \otimes \mathrm{id})^{*}:\left[k[G / H] \otimes S^{n}, R\right]_{k[G]}^{\oplus} \longrightarrow\left[k[G / K] \otimes S^{n}, R\right]_{k[G]}^{\oplus}
$$

and this completes the proof.

Lemma 3.5. Let $T$ be a $G$-set and let $n$ be a non-negative integer. Then $\omega_{i}\left(k[T] \otimes S^{n}\right)=0$ if $i \neq n$ and $\omega_{n}\left(k[T] \otimes S^{n}\right) \cong \overline{k[T]}$ as Hecke functors over $k$.

Proof. By Lemma 3.2, we see that $\left(k[T] \otimes S^{n}\right)^{H} \cong k[T]^{H} \otimes S^{n}$. Hence the lemma follows from the classical Dold-Thom theorem [3].

Lemma 3.6. Let $f: A \rightarrow B$ be a morphism in $\mathbb{M}_{k[G]}$. Then there are morphisms $h: F \rightarrow A$ and $q: B \rightarrow C$ in $\mathbb{M}_{k[G]}$ such that $F \rightarrow A \rightarrow B \rightarrow C$ is a sequence of homotopy theoretical G-fibrations.

Proof. Let $\Omega B \stackrel{i}{\longrightarrow} P B \stackrel{a}{\longrightarrow} B$ be the usual path fibration with $0 \in B$ as a base point. It is clear that $\Omega B$ and $P B$ are objects of $\boldsymbol{M}_{k\lfloor G]}$ in the obvious way, and $i$ and $\pi$ are morphisms of $\boldsymbol{M}_{k[G]}$. Let $F$ be the fibre product of $f$ and $\pi$, then clearly $F$ is the required one. Note that the natural map $\delta: \Omega B \rightarrow F$ is also a morphism of $\mathbb{M}_{\left.k_{i G G}\right]}$. Next let $C$ be the fibre of the morphism $f \otimes$ id $: A \otimes S^{1} \rightarrow B \otimes S^{1}$ in the above sense. We note that the canonical $G$-homotopy equivalence $j: B \rightarrow \Omega\left(B \otimes S^{1}\right)$ is also a morphism of $\boldsymbol{M}_{k[G]}$. Let $q$ be the composite $B \stackrel{j}{\longrightarrow} \Omega\left(B \otimes S^{1}\right) \stackrel{\delta}{\longrightarrow} C$. Then $A \stackrel{f}{\longrightarrow} B \stackrel{q}{\longrightarrow} C$ is clearly a homotopy theoretical $G$-fibration. 
As usual $F$ is called a fibre of $f$, and by abuse of language $C$ is called a cofibre of $f$.

Proposition 3.7. Let $A$ be a Hecke functor and let $n$ be a nonnegative integer. Then there is a topological $k[G]$-module $R$ such that $\omega_{i}(R)=0$ if $i \neq n$ and $\omega_{n}(R) \cong A$ as Hecke functors over $k$.

Proof. For a Hecke functor A there is a projective resolution

$$
0 \longleftarrow A \longleftarrow \overline{k\left[T_{0}\right]} \stackrel{\alpha_{0}}{\longleftarrow} \overline{k\left[T_{1}\right] \longleftarrow} \cdots
$$

by Proposition 2.3. Also we have isomorphisms $\operatorname{Mor}\left(\overline{k\left[T_{1}\right]}, \overline{k\left[T_{0}\right]}\right) \cong$ $\overline{k\left[T_{0}\right]}\left(T_{1}\right) \cong \operatorname{Hom}_{k[G]}\left(k\left[T_{1}\right], k\left[T_{0}\right]\right)$. Hence a morphism $\alpha_{0}$ is represented by a $k[G]$-homomorphism $f_{0}: k\left[T_{1}\right] \longrightarrow k\left[T_{0}\right]$. Consider the continuous $k[G]$-homomorphism $f_{0} \otimes \mathrm{id}: k\left[T_{1}\right] \otimes S^{n} \longrightarrow k\left[T_{0}\right] \otimes S^{n}$. By Lemma 3.6 we obtain a $G$-homotopy theoretical fibration

$$
k\left[T_{1}\right] \otimes S^{n} \longrightarrow k\left[T_{0}\right] \otimes S^{n} \longrightarrow X_{1} .
$$

By Lemma 3.5 we easily see that $\omega_{i}\left(X_{1}\right)=0$ for $i<n, \omega_{n}\left(X_{1}\right) \cong A$ and $\omega_{n+1}\left(X_{1}\right) \cong \operatorname{Ker}\left(\alpha_{0}\right)$ as Hecke functors. Choose a $G$-set $T_{2}$ and an epimorphism $\alpha_{1}: \overline{k\left[T_{2}\right]} \rightarrow \omega_{n+1}\left(X_{1}\right)$. By Proposition 2.3, we have $\alpha_{1} \in$ $\omega_{n+1}\left(X_{1}\right)\left(T_{2}\right)$, and hence $\alpha_{1}$ is represented by a continuous $k[G]-$ homomorphism

$$
f_{1}: k\left[T_{2}\right] \otimes S^{n+1} \longrightarrow X_{1} .
$$

Let $X_{2}$ be the cofibre of $f_{1}$. By Lemma 3.5 we see that $\omega_{i}\left(X_{2}\right)=0$ for $i<n$ and $i=n+1, \omega_{n}\left(X_{2}\right) \cong A$ and $\omega_{n-2}\left(X_{2}\right) \cong \operatorname{Ker}\left(\alpha_{1}\right)$. Iterating this construction we obtain topological $k[G]$-modules $X_{k}$ and continuous $k[G]$-homomorphisms $X_{k} \rightarrow X_{k+1}$. Put $R=\lim _{\longrightarrow} X_{k}$, then $R$ satisfies the required property.

The $G$-space $R$ in the proposition is called an Eilenberg-MacLane $G$-space (EMG-space for short) of the type $(\mathrm{A}, n)$. In [1] it is shown that for any Bredon functor $B$ and an integer $n$, there is an $E M G$-space $K(B, n)$.

Let $M$ be a module valued Bredon functor and let $X$ be a $G_{-}^{-}$ CW complex. Then Bredon [1] has defined the ordinary $G$-cohomology theory $H_{G}^{n}(X ; M)$ and has shown a natural isomorphism

$$
H_{G}^{n}(X ; M) \cong[X, K(M, n)]_{G} \text {. }
$$


One can regard $H_{G}^{n}(X ; M)$ as a Bredon functor by $H_{G}^{n}(X ; M)$ $(G / H)=H_{G}^{n}(G / H \times X ; M)$. It is known [7] that if $M$ is a Mackey functor then one can define an $R O(G)$-graded ordinary $G$-cohomology theory $H_{G}^{*}(X ; M)$ which can be made into a Mackey functor as above. For Hecke functors by Proposition 3.7 we easily obtain

Proposition 3.8. Let $M$ be a Hecke functor. Then so is $H_{G}^{n}(X$; M) for any $n \in Z$.

\section{§4. Equivariant Dold-Thom Theorem}

In the previous section we have shown that a Hecke functor over $k$ is given as a Bredon homotopy group of a topological $k[G]$-module. In this section on the other hand we show how a topological $k[G]-$ module is characterized by means of Hecke functors. In the abelian category of Hecke functors over $k$, consider a chain complex of Hecke functors over $k$. We denote by $\partial \mathbf{H e c}_{k}^{G}$ the category of chain complexes of Hecke functors over $k$ and chain maps. To a chain complex assigning its $n$-dimensional homology, we obtain functor

$$
H_{n}: \partial \mathbf{H e c}_{k}^{G} \longrightarrow \mathbf{H e c}_{k}^{G} \text {. }
$$

A chain map $f: C_{*} \rightarrow C_{*}^{\prime}$ is called a weak equivalence if the induced homomorphism

$$
f_{*}: H_{n}\left(C_{*}\right) \longrightarrow H_{n}\left(C_{*}^{\prime}\right)
$$

is an isomorphism for any $n$. We denote by $\mathrm{Ho}\left(\partial \mathbf{H e c}_{k}^{G}\right)$ the associated homotopy category, i. e., the category localized with respect to weak equivalences.

Next let $R$ and $Q$ be topological $k[G]$-modules. A morphism $f: R \rightarrow Q$ in $\boldsymbol{M}_{k[G]}$ is called a weak equivalence if the induced homomorphism

$$
f_{*}: \omega_{n}(R) \longrightarrow \omega_{n}(Q)
$$

is an isomorphism for any $n$. We denote by $\mathrm{Ho}\left(\boldsymbol{M}_{k[G]}\right)$ the associated homotopy category. Then our main result is

Theorem 4.1. There are equivalences of categories

$$
\mathrm{Ho}\left(\boldsymbol{M}_{k[G]}\right) \stackrel{\Phi}{\underset{\Psi}{\leftrightarrows}} \mathrm{Ho}\left(\partial \mathrm{Hec}_{k}^{G}\right)
$$


such that $H_{n} \circ \Phi=\omega_{n}$ and $\omega_{n} \circ \Psi=H_{n}$ for any $n$.

To prove the theorem we recall some results of Quillen [10]. Since $\mathbf{H e c}_{k}^{G}$ is an abelian category with enough projectives, the category $\partial \mathrm{Hec}_{k}^{G}$ is a model category in which weak equivalences are defined as above, a fibration is an epimorphism and a cofibration $f: C_{*} \rightarrow C_{*}^{\prime}$ is a chain map such that $\operatorname{Cok}(f)$ is a projective chain complex. Therefore a projective chain complex is a fibrant-cofibrant object. A projective chain complex of the form

$$
\longrightarrow \overline{k\left[T_{i}\right]} \longrightarrow \overline{k\left[T_{i-1}\right]} \rightarrow \cdots \rightarrow \overline{k\left[T_{0}\right]}
$$

is called representable where $T_{i}$ is a $G$-set. Let $\mathbb{P}$ and $\boldsymbol{R}$ be full subcategories of $\partial \mathrm{Hec}_{k}^{G}$ consisting of projective objects and representable objects, respectively. By taking chain homotopy classes of chain maps we obtain quotient categories $\pi \boldsymbol{P}$ and $\pi \boldsymbol{R}$. It is well known [10] that there is an equivalence of categories

$$
\gamma: \pi \boldsymbol{P} \longrightarrow \mathrm{Ho}\left(\partial \mathbf{H e c}_{k}^{G}\right) \text {. }
$$

It is easy to show that the inclusion $\pi \boldsymbol{R} \rightarrow \pi \boldsymbol{P}$ is an equivalence. Therefore Theorem 4.1 can be stated as follows.

Theorem 4.2. There are equivalences of categories

$$
\mathrm{Ho}\left(\boldsymbol{M}_{k[G]}\right) \stackrel{\Phi^{\prime}}{\rightleftarrows} \pi \boldsymbol{R}
$$

such that $H_{n} \circ \Phi^{\prime}=\omega_{n}$ and $\omega_{n} \circ \Psi^{\prime}=H_{n}$ for any $n$.

Proof. First we construct a functor $\Phi^{\prime}$. Given $R \in \boldsymbol{M}_{k[G]}$, we define $R_{i} \in \boldsymbol{M}_{k[G]}$ and $k\left[T_{i}\right]$ inductively as follows. Let $R_{0}=R$ and suppose that $R_{q}$ and $k\left[T_{q-1}\right]$ have been defined. Choose a $G$-set $T_{q}$ and an epimorphism

$$
a_{q}: \overline{k\left[T_{q}\right]} \longrightarrow \omega_{0}\left(R_{q}\right)
$$

by Proposition 2.3 and Proposition 3.4. Then by Proposition $2.3 a_{q}$

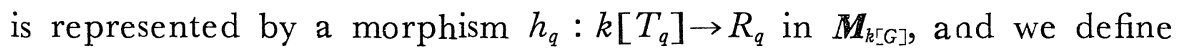
$R_{q+1}$ to be the homotopy theoretical fibre of $h_{q}$. Let $d_{q}$ be the composite

$$
\omega_{0}\left(k\left[T_{q}\right]\right) \longrightarrow \omega_{0}\left(R_{q}\right) \longrightarrow \omega_{0}\left(k\left[T_{q-1}\right]\right)
$$

then $C_{R}=\left\{\overline{k\left[T_{q}\right]}, d_{q}\right\}$ is clearly a representable chain complex and 
$H_{n}\left(C_{R}\right) \cong \omega_{n}(R)$ for any $n$.

Let $f: R \rightarrow Q$ be a morphism in $\mathbb{M}_{k[G]}$. Choose $C_{R}=\left\{\overline{k\left[T_{q}\right]}, d_{q}\right\}$ and $C_{Q}=\left\{\overline{k\left[S_{q}\right]}, d_{q}^{\prime}\right\}$ as above. Consider the diagram

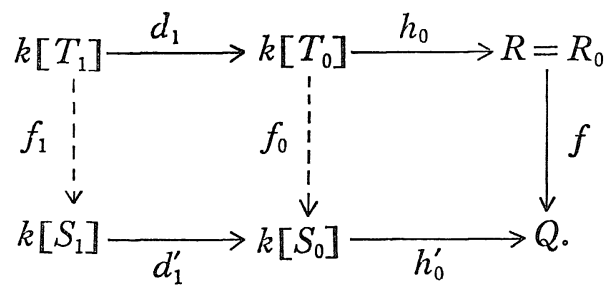

Then by Proposition 2.3, there is a dotted morphism $f_{0}$ such that the right-hand square is homotopy commutative in category $\boldsymbol{M}_{k[G]}$. Then by Lemma 3.6 we see that there is a dotted morphism $f_{1}$ and the left-hand square is also homotopy commutative in $\boldsymbol{M}_{k[G]}$. Iterating this we obtain a chain map

$$
f_{\sharp}: C_{R} \longrightarrow C_{Q}
$$

such that $\left(f_{\sharp}\right)_{*}: H_{n}\left(C_{R}\right) \rightarrow H_{n}\left(C_{Q}\right)$ is equal to $f_{*}: \omega_{n}(R) \rightarrow \omega_{n}(Q)$. Moreover one can easily see that $f_{\sharp}$ is unique up to chain homotopy. Now note that if $f: R \rightarrow Q$ is a weak equivalence, then clearly so is $f_{\sharp}$, and in $\pi \boldsymbol{R}$ a weak equivalence is a chain equivalence. Hence assigning $C_{R}$ to $R$, we obtain a functor

$$
\Phi^{\prime}: \mathrm{Ho}\left(\boldsymbol{M}_{k[G]}\right) \longrightarrow \pi \mathbb{R} .
$$

Next we define $\Psi^{\prime}$. Let a chain complex $C$

$$
\longrightarrow \overline{k\left[T_{i}\right]} \stackrel{d_{i}}{\longrightarrow} \overline{k\left[T_{i-1}\right]} \rightarrow \cdots \rightarrow \overline{k\left[T_{1}\right]} \stackrel{d_{1}}{\longrightarrow} \overline{k\left[T_{0}\right]}
$$

be given. Let $\alpha_{i}: k\left[T_{i}\right] \rightarrow k\left[T_{i-1}\right]$ be a representing $k[G]$-map. Define $R_{i} \in M_{k[G]}$ and a morphism $q_{i}: k\left[T_{i+1}\right] \otimes S^{i} \rightarrow R_{i}$ inductively as follows. Let $R_{0}=k\left[T_{0}\right]$ and $q_{0}=\alpha_{1}$. Suppose that $R_{i}$ and $q_{i}$ are defined for $i<l$. Then let $R_{\ell}$ be the cofibre of $q_{\ell-1}$. Then by taking $\omega_{\ell-1}$ we obtain a commutative diagram

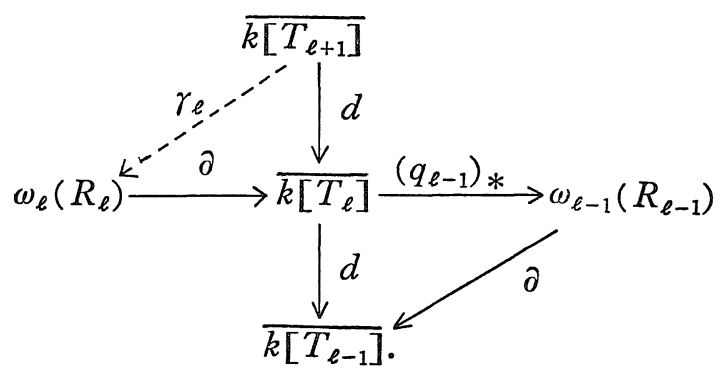


By induction we see that $\omega_{j}\left(R_{i}\right)=0$ if $j>i$, and hence the homomorphisms $\partial$ in the diagram are injective. Therefore there is a unique map $\gamma_{\ell}$ such that $\partial \circ \gamma_{\ell}=d$. Then define $q_{\ell}: k\left[T_{\ell+1}\right] \otimes S^{\ell} \rightarrow R_{\ell}$ to be the morphism in $\boldsymbol{M}_{k[G]}$ representing $r_{\ell}$. Now we define

$$
R_{C}=\lim _{\longrightarrow} R_{\ell}
$$

Note that in the definition of $R_{\ell}$ all constructions are done in a functorial way and hence we obtain a functor $\boldsymbol{R} \rightarrow \boldsymbol{M}_{k[G]}$. It is clear that $\omega_{n}\left(R_{C}\right) \cong H_{n}(C)$ for any $n$. Hence we have a functor

$$
\Psi^{\prime}: \mathrm{Ho}(\boldsymbol{R}) \longrightarrow \mathrm{Ho}\left(\boldsymbol{M}_{k[G]}\right) \text {. }
$$

But we replace $\mathrm{Ho}(\boldsymbol{R})$ with $\pi(\boldsymbol{R})$ by equivalence and we obtain a required functor. Now it is easy to see that $\Phi^{\prime}$ is an equivalence of categories inverse to $\Psi^{\prime}$. This completes the proof.

Next we consider a special case that the ground ring $k$ is a PID such that $k \ni 1 /|G|$, and show that an equivariant version of DoldThom theorem holds in this case.

Lemma 4. 3. Let $k$ be as above. Then any $k[G]$-submodule of a projective $k[G]-$ module is projective.

Proof. Let $K$ be the quotient field of $k$. Let $V$ be a $k[G]-$ module free as a $k$-module. Then it is easy to see that $V$ is $k[G]$ free if and only if $V \otimes K$ is $K[G]$-free. Now to prove the lemma it suffices to show that if $M$ is a $k[G]$-submodule of a free $k[G]$-module $F$, then $M$ is projective. Note that $M$ is then $k$-free. It is obvious that the inclusion $M \otimes K \rightarrow F \otimes K$ splits as $K[G]$-modules. Hence there is a $K[G]$-module $W$ and $(M \otimes K) \oplus W \cong F \otimes K$. Choose a $k[G]-$ lattice $L$ of $W$ so that $W \cong L \otimes K$. Then we have

$$
(M \oplus L) \otimes K \cong F \otimes K
$$

and hence $M \oplus L$ is $k[G]$-free. This completes the proof.

Proposition 4.4. Let $k$ be as above. Then any sub Hecke functor over $k$ of a projective Hecke functor over $k$ is projective.

Proof. Let $T$ be a $G$-set and let $A$ be a sub Hecke functor of 
$\overline{k[T]}$. It suffices to show that $A(G / e)$ is a projective $k[G]$-module and $A \cong \overline{A(G / e)}$. By Lemma 4.3,A(G/e) is projective. Let $H$ be a subgroup of $G$ and let $\pi: G=G / e \rightarrow G / H$ be the projection. It is clear that $\pi^{*}: A(G / H) \rightarrow A(G / e)$ maps $A(G / H)$ into $A(G / e)^{H}$. The inclusion $A \rightarrow \overline{k[T]}$ is then factored as $A \stackrel{i}{\longrightarrow} \overline{A(G / e)} \longrightarrow \overline{k[T]}$ and clearly $i$ is a monomorphism. Let $B=\overline{A(G / e)} / A$ be the quotient Hecke functor. Since $B$ is cohomological, $B(G / e)=0$ and $k \ni 1 /|G|$, we easily see that $B \cong 0$. This shows that $A \cong \overline{A(G / e)}$.

Let $\boldsymbol{A}$ be an abelian category with enough projectives and suppose that any subobject of a projective object is projective. Let $\partial \boldsymbol{P}$ be the category of projective chain complexes in $\boldsymbol{A}$ and let $\pi(\partial \boldsymbol{P})$ be the quotient category as defined before. Then it is well known that the functor

$$
H_{*}: \pi(\partial \boldsymbol{P}) \longrightarrow \boldsymbol{A}_{*}
$$

is an equivalence of categories where $\boldsymbol{A}_{*}$ is the category of nonnegatively graded objects in $\boldsymbol{A}$. Then combining Theorem 4.1 and Proposition 4.4 we have

Theorem 4.5. Let $k$ be a PID such that $1 /|G| \in k$. Then the Bredon homotopy groups

$$
\omega_{*}: \mathrm{Ho}\left(\boldsymbol{M}_{k[G]}\right) \longrightarrow\left(\mathbf{H e c}_{k}^{G}\right)_{*}
$$

is an equivalence of categories.

Corollary 4.6. Let $k$ be as above. Then a topological $k[G]-$ module has a G-homotopy type of a generalized EMG-space.

Now if $G$ is trivial group and $k=Z$, then Theorem 4.5 is the wellknown Dold-Thom theorem. For general $G$ and $k$, Theorem 4.5 does not hold as the following example shows.

Example 4. 7. Let $G=\boldsymbol{Z}_{2}$ and $k=\boldsymbol{Z}$. Recall that $\operatorname{Mor}\left(\overline{\boldsymbol{Z}\left[\boldsymbol{Z}_{2}\right]}\right.$, $\overline{\boldsymbol{Z}[1]}) \cong \operatorname{Hom}_{\boldsymbol{Z}\left[\boldsymbol{Z}_{2}\right]}\left(\boldsymbol{Z}\left[\boldsymbol{Z}_{2}\right], \boldsymbol{Z}\right) \cong \boldsymbol{Z}$. Let $d: \overline{\boldsymbol{Z}\left[\boldsymbol{Z}_{2}\right]} \rightarrow \overline{\boldsymbol{Z}[1]}$ be the morphism corresponding a generator of $\boldsymbol{Z}$. we regard

$$
C_{1}=\overline{\boldsymbol{Z}\left[\boldsymbol{Z}_{2}\right]} \stackrel{d}{\longrightarrow} C_{0}=\overline{\boldsymbol{Z}[1]}
$$


as a projective chain complex $C_{*}$. Let $R=\Psi^{\prime}\left(C_{*}\right)$ be a topological $\boldsymbol{Z}\left[\boldsymbol{Z}_{2}\right]$-module as in Theorem 4.2. Then one can easily show that

$$
R \not K\left(H_{0}\left(C_{*}\right), 0\right) \times K\left(H_{1}\left(C_{*}\right), 1\right) .
$$

\section{§5. A Remark on a Relation to Mackey Functors}

Let $R$ be a topological $G$-module with a $G$-homotopy type of a $G$-CW complex. Consider the $G$-spectrum $\mathbb{R}=\left\{R \otimes \Sigma^{V}\right\}_{V}$, where $V$ is a real representation of $G$ and $\Sigma^{V}$ is the one point compactification of $V$. It is known [7] that $\boldsymbol{R}$ is an $\Omega-G$-spectrum and hence $R$ is an infinite loop $G$-space. Therefore the stable $G$-homotopy group $\boldsymbol{\omega}_{*}^{\boldsymbol{S}}(\boldsymbol{R})$ can be defined as a Mackey functor (over $\mathbb{Z}$ ). It is easy to see that this Mackey functor coincides with the underlying Mackey functor of the Hecke functor $\omega_{*}(R)$. Let $M$ be a Mackey functor, then it is known [8] that an $E M G$-space $K(M, n)$ is an infinite loop $G$-space. Then by Proposition 3.7 we easily obtain.

Proposition 5.1. A Mackey functor $M$ is a Hecke functor if and only if an EMG-space $K(M, n)$ is G-homotopy equivalent to a topological G-module as infinite loop G-spaces for any $n$.

Corollary 5. 2. There is an EMG-space $K(B, n)$ which is a $G$ homotopy type of an infinite loop $G$-space but not a G-homotopy type of a topological G-module.

Proof. It is enough to show that there is a Bredon functor $B$ which extends to a Mackey functor but does not extend to a Hecke functor. Let $G=\mathbb{Z}_{2}$. Let $\Omega$ be the Burnside functor, i. e., $\Omega(G / H)=$ $A(H)$, the Burnside ring of $H$. It is well known that $\Omega$ is a Mackey functor and let $B$ be the underlying Bredon functor. Let $\pi: \mathbb{Z}_{2} \rightarrow$ $\boldsymbol{Z}_{2} / \boldsymbol{Z}_{2}$ be the projection, then $\pi^{*}: B\left(\boldsymbol{Z}_{2} / \mathbb{Z}_{2}\right)=A\left(\boldsymbol{Z}_{2}\right) \rightarrow B\left(\mathbb{Z}_{2}\right)=\mathbb{Z}$ is the augmentation homomorphism. Suppose that $B$ extends to a Hecke functor or equivalently to a cohomological Mackey functor (Theorem 2. 2). Then we have a homomorphism $\pi_{*}: \mathbb{Z} \rightarrow A\left(\mathbb{Z}_{2}\right)$ such that $\pi_{*} \circ \pi^{*}=2$, but this is clearly impossible and this completes the proof. 


\section{References}

[1] Bredon, G., Equivariant homology theories, Lecture Notes in Math., 34, Springer-Verlag, 1967.

[2] tom Dieck, T., Equivariant homology and Mackey functor, Math. Ann., 206 (1973) 67-78.

[3] Dold, A., and Thom, R., Quasifaserungen und unendliche symmetrische Produkte, Ann. of Math., 67 (1958), 239-281.

[4] Dress, A., Contribution to the theory of induced representations, Lecture Notes in Math., 342, Springer-Verlag, 1973.

[5] Green, J. A., Axiomatic representation theory for finite groups, J. of Pure and Applied Algebra, 1 (1971), 41-77.

[6] Kosniowski, C., Equivariant cohomology and stable cohomotopy, Math. Ann., 210 (1974).

[7] Lewis, G., May, J. P., McClure, J. and Steinberger, M., Equivariant stable homotopy theory, Springer Lecture Notes (to appear)

[8] Nishida, G., Mackey functors and equivariant generalized cohomology theories, to appear.

[9] , The transfer homomorphism in equivariant generalized cohomology theories, J. Math. Kyoto Univ., 18 (1978), 435-451.

[10] Quillen, D., Homotopical algebra, Lecture Notes in Math., 28, Springer-Verlag, 1972.

[11] Yoshida, T., On G-functors II; Hecke operators and G-functors, preprint. 
